\title{
A FINITE RECURSION FORMULA FOR THE COEFFICIENTS IN ASYMPTOTIC EXPANSIONS ${ }^{1}$
}

BY

T. D. RINEY

1. Introduction. In recent years the asymptotic behavior in the neighborhood of infinity of entire functions, $G(z)$, which are defined by their power series developments

$$
G(z)=\sum_{n=0}^{\infty} g(n) z^{n},
$$

have been studied by W. B. Ford [2], E. M. Wright $[9 ; 10 ; 11]$, H. K. Hughes $[3 ; 4]$, and J. H. B. Kemperman [6]. To apply their theory it is required that the coefficient $g(n)$ may be considered as a function $g(w)$ of a complex variable and as such admits an asymptotic factorial expansion in every right halfplane. From their investigations it follows that the constants occurring in this asymptotic expansion of $g(w)$ are precisely the constants which occur in the asymptotic expansion of $G(z)$.

It is known from a lemma which appears in $[2 ; 4 ; 11]$ that the function

$$
{ }_{p} g_{q}(w)=\prod_{i=1}^{p} \Gamma\left(w+\sigma_{i}\right) / \sum_{j=0}^{q} \Gamma\left(w+\rho_{j}\right)
$$

admits the required factorial expansion. Here $p$ and $q$ are non-negative integers with $p \leqq q$; the $\sigma_{i}$ and $\rho_{j}$ denote arbitrary complex parameters. The complete determination of the asymptotic expansion for large $|z|$ of the important class of functions

$$
{ }_{p} G_{q}(z)=\sum_{n=0}^{\infty}{ }_{p} g_{q}(n) z^{n}
$$

is thus dependent upon a procedure for computing the constants occurring in the expansion of ${ }_{p} g_{q}(w)$. In an earlier paper [7] the fact that ${ }_{p} g_{q}(w)$ satisfies a first order difference equation was used to obtain an inductive formula for the coefficients depending on all the previously calculated coefficients.

This paper deals with a simpler formula for calculating these coefficients. For this purpose we introduce a function $\phi(t)$ defined as an inverse transform

Received by the editors November 10, 1956.

(1) Included in a thesis submitted to the Graduate School of Purdue University in partial fulfillment of the requirements for the degree of Doctor of Philosophy. The work was directed by Professor H. K. Hughes and J. H. B. Kemperman. 
of ${ }_{p} g_{q}(w)$ and use the difference equation to obtain a differential equation of Fuchsian type satisfied by $\phi(t)$. The expansion of $\phi(t)$ about one of the regular singular points of the differential equation will be determined and shown to generate the constants in question. By this procedure we shall obtain a finite recursion formula of fixed length $q$ for the coefficients.

2. The factorial expansion. The lemma cited in $\S 1$ is now stated as a theorem. The proof which is based on Stirling's formula is omitted.

THEOREM 2.1. Let h be any real number and let $M$ be any non-negative integer. Then in the half-plane $\operatorname{Re}(w)>-h$ with $|w|$ large, ${ }_{p} g_{q}(w)$ admits the asymptotic development

$$
{ }_{p} g_{q}(w)=\left((2 \pi)^{1 / 2}\right)^{1-\alpha} \alpha^{\alpha w+\beta-1 / 2}
$$

$$
\cdot\left[\sum_{m=0}^{M} \frac{c_{m}}{\Gamma(\alpha w+\beta+m)}+O\left(\frac{1}{\Gamma(\alpha w+\beta+M+1)}\right)\right],
$$

where $c_{0}=1$ and

$$
\alpha=q+1-p ; \quad \beta=\sum_{j=0}^{q} \rho_{j}-\sum_{i=0}^{p} \sigma_{i}+(1-\alpha) / 2 .
$$

In the subsequent investigation it is convenient to introduce the auxiliary function

$$
H(x)=\left((2 \pi)^{1 / 2}\right)^{\alpha-1} \alpha^{-x-q-5 / 2} \Gamma(x){ }_{p} g_{q}\left(\frac{x-\beta+q+3}{\alpha}\right),
$$

where $x$ is a complex argument. From the multiplication theorem of Gauss [8, p. 240] it follows that $H(x)$ may be written in the form

$$
H(x)=\alpha^{-q-3}{ }_{p} g_{q}\left(\frac{x-\beta+q+3}{\alpha}\right) \prod_{r=0}^{\alpha-1} \Gamma\left(\frac{x+r}{\alpha}\right) .
$$

We note that as long as $x$ is confined to an arbitrary right half-plane,

$$
\Gamma(x) / \Gamma(x+a) \sim x^{-a} \text { for }|x| \text { large. }
$$

This is true for any complex number $a$ by Stirling's formula. It therefore follows from (2.4), (1.1) and (2.2) that if $x$ is restricted to any right half-plane then

$$
H(x) \sim x^{-q-3} \text { for }|x| \text { large. }
$$

Moreover, it follows from (2.3) and Theorem 2.1 that in any right half-plane $\operatorname{Re}(x)>-h, H(x)$ has the asymptotic development

$$
H(x)=\sum_{m=0}^{M} \frac{c_{m} \Gamma(x)}{\Gamma(x+q+3+m)}+O\left(\frac{\Gamma(x)}{\Gamma(x+q+4+M)}\right) .
$$


3. Definition of $\phi(t)$. We introduce the notation

$$
\begin{aligned}
\lambda_{0} & =\max \left\{0, \max _{i} \operatorname{Re}\left(\beta-q-3-\alpha \sigma_{i}\right)\right\}, \\
\lambda & =\text { any real number greater than } \lambda_{0} .
\end{aligned}
$$

It follows from (2.3) and (1.1) that $H(x)$ is single-valued and regular in the half-plane $\operatorname{Re}(x)>\lambda_{0}$. From (2.5) we have $H(x)=O\left(x^{-q-3}\right)$ for $x$ in any right half-plane and $|x|$ large. Hence for real values $\xi$ the inversion integral

$$
U(\xi)=\frac{1}{2 \pi i} \int_{\lambda-i \infty}^{\lambda+i \infty} e^{\xi x} H(x) d x
$$

converges. It follows from a well-known theorem [1, p. 126] that $H(x)$ is represented by the Laplacian integral

$$
H(x)=\int_{0}^{\infty} e^{-\xi x} U(\xi) d \xi,
$$

provided $\operatorname{Re}(x)>\lambda_{0}$. Making the transformation $\xi=\log t^{-1}, t=e^{-\xi}$, these two relations become

$$
\phi(t)=\frac{1}{2 \pi i} \int_{\lambda-i \infty}^{\lambda+i \infty} t^{-x} H(x) d x, \quad 0<t \leqq 1,
$$

and

$$
H(x)=\int_{0}^{1} t^{x-1} \phi(t) d t, \quad \operatorname{Re}(x)>\lambda_{0},
$$

where we have replaced $U\left(\log t^{-1}\right)$ by $\phi(t)$.

We now derive upper bounds for $\phi(t)$ at both $t=1$ and $t=0+$.

Theorem 3.1. The function $\phi(t)$ defined by (3.2) is $O\left(t^{-\lambda}\right)$ as $t \rightarrow 0+$, where $\lambda$ is defined by (3.1). Moreover, $\phi(t)=O\left((1-t)^{q+2}\right)$ near $t=1$.

Proof. Let $v$ be real and set $x=\lambda+i v$, then

$$
\phi(t)=\frac{1}{2 \pi} \int_{-\infty}^{\infty} t^{-\lambda-i v} H(\lambda+i v) d v .
$$

From (2.5) there exists a constant $M$ such that

$$
|H(\lambda+i v)|<M\left(\lambda^{2}+v^{2}\right)^{-(q+3) / 2}
$$

for $|\lambda+i v|$ large enough, $\lambda>\lambda_{0}$. Since $H(\lambda+i v)$ has no poles for $\lambda>\lambda_{0}$ it follows that (3.4) holds, for $\lambda \geqq \lambda_{0}+\epsilon$ (for any $\epsilon>0$ ) with $M$ independent of $\lambda$ and $v$. Thus 


$$
|\phi(t)|<\frac{M}{\pi} t^{-\lambda} \int_{0}^{\infty}\left(\lambda^{2}+v^{2}\right)^{-(q+3) / 2} d v
$$

We set $v^{2}=\lambda^{2} u$ and obtain

$$
|\phi(t)|<\frac{M}{2 \pi} \lambda^{-q-2} t^{-\lambda} \int_{0}^{\infty}(u+1)^{-(q+3) / 2} u^{-1 / 2} d u,
$$

from which the first assertion of the theorem follows since the integral converges. If we choose $\lambda=(1-t)^{-1}$ in the latter inequality we see that near $t=1$

$$
\phi(t)=O\left((1-t)^{q+2}\right)
$$

since $t^{-\lambda}$ is bounded for this special choise of $\lambda$.

4. A differential equation satisfied $\phi(t)$. The reader will readily note that by applying the recurrence relation $\Gamma(x+1)=x \Gamma(x)$ to (2.4) we immediately obtain the difference equation

$$
P(x) H(x)=Q(x+\alpha) H(x+\alpha),
$$

where

$$
\begin{aligned}
& P(x)=\prod_{i=1}^{p}\left(x-\beta+q+3+\alpha \sigma_{i}\right) \prod_{r=0}^{\alpha-1}(x+r), \\
& Q(x)=\prod_{j=0}^{q}\left(x-\alpha-\beta+q+3+\alpha \rho_{j}\right) .
\end{aligned}
$$

Equations (3.2) and (4.1) may be combined to yield a linear differential equation satisfied by $\phi(t)$.

Theorem 4.1. Let $\theta=t(d / d t)$, then the function $\phi(t)$ defined by (3.2) is an integral of the linear differential equation

$$
L(y) \equiv\left\{t^{\alpha} Q(-\theta)-P(-\theta)\right\} y(t)=0,
$$

where $P(x)$ and $Q(x)$ are defined by (4.2).

Proof. The integral (3.2) which defines $\phi(t)$ may be differentiated $m(m \leqq q+1)$ times under the integral sign. To show this it suffices to show that

$$
\int_{\lambda-i \infty}^{\lambda+i \infty}(-\theta)^{m} t^{-x} H(x) d x=\int_{\lambda-i \infty}^{\lambda+i \infty} t^{-x} x^{m} H(x) d x
$$

is uniformly convergent. This is indeed true for $m \leqq q+1$ as follows from (2.5). Observing that

$$
P(-\theta) t^{-x}=P(x) t^{-x} \text { and } Q(-\theta) t^{-x}=Q(x) t^{-x},
$$

we have 


$$
\begin{aligned}
P(-\theta) \phi(t) & =\frac{1}{2 \pi i} \int_{\lambda-i \infty}^{\lambda+i \infty} P(-\theta) t^{-x} H(x) d x \\
& =\frac{1}{2 \pi i} \int_{\lambda-i \infty}^{\lambda+i \infty} P(x) t^{-x} H(x) d x \\
& =\frac{1}{2 \pi i} \int_{\lambda-i \infty}^{\lambda+i \infty} Q(x+\alpha) t^{-x} H(x+\alpha) d x \\
& =\frac{t^{\alpha}}{2 \pi i} \int_{\lambda+\alpha-i \infty}^{\lambda+\alpha+i \infty} Q(x) t^{-x} H(x) d x \\
& =t^{\alpha} Q(-\theta) \phi(t)
\end{aligned}
$$

which proves the assertion.

In order to write $L(y)=0$ in the usual linear form we shall need the following lemma.

Lemma 4.1. Let $\theta=t d / d t$ and let $B_{m}(m=0,1, \cdots, q)$ be arbitrary complex parameters. Then the constants $A_{k}$ in the operator identity

$$
\prod_{m=0}^{q}\left(\theta+B_{m}\right)=\sum_{k=0}^{q+1} A_{k} t^{k} \frac{d^{k}}{d t^{k}}
$$

are given recursively by the formula

$$
A_{k}=\frac{1}{k !} \prod_{m=0}^{q}\left(k+B_{m}\right)-\sum_{j=0}^{k-1} \frac{A_{j}}{(k-j) !} .
$$

In particular,

$$
A_{q+1}=1 ; \quad A_{q}=\sum_{m=0}^{q} B_{m}+q(q+1) / 2 .
$$

Proof. It is clear that such constants exist. Observing that

$$
\prod_{m=0}^{q}\left(\theta+B_{m}\right) t^{x}=t^{x} \prod_{m=0}^{q}\left(x+B_{m}\right)
$$

and

$$
t^{k} \frac{d^{k}}{d t^{k}} t^{x}=\frac{\Gamma(x+1)}{\Gamma(x+1-k)} t^{x}
$$

we have the identity

$$
\prod_{m=0}^{q}\left(x+B_{m}\right)=\sum_{k=0}^{q+1} A_{k} \frac{\Gamma(x+1)}{\Gamma(x+1-k)},
$$

or 


$$
\frac{1}{\Gamma(x+1)} \prod_{m=0}^{q}\left(x+B_{m}\right)=\sum_{j=0}^{q+1} \frac{A_{j}}{\Gamma(k+1-j)} .
$$

We put $x=k$ in (4.7) and obtain

$$
\frac{1}{k !} \prod_{m=0}^{q}\left(k+B_{m}\right)=\sum_{j=0}^{k} \frac{A_{j}}{\Gamma(x-j+1)},
$$

from which (4.5) follows. Relations (4.6) follow from a direct operation of $\prod_{m=0}^{m=q}\left(\theta+B_{m}\right)$ on a function $y(t)$.

Theовем 4.2. The differential equation $L(y)=0$, of which $\phi(t)$ is an integral, may be written in the form

$$
L(y) \equiv \sum_{k=\alpha}^{q+1} t^{k-\alpha}\left(E_{k} t^{\alpha}-C_{k}\right) y^{(k)}(t)+\sum_{k=0}^{\alpha-1} E_{k} t^{k} y^{(k)}(t)=0,
$$

where

$$
E_{k}=\frac{1}{k !} \prod_{m=0}^{q}\left(\beta+k-p-2-\alpha \rho_{m}\right)-\sum_{j=0}^{k-1} \frac{E_{j}}{(k-j) !}(0 \leqq k \leqq q+1),
$$

and

$$
\begin{array}{r}
C_{k}=\frac{1}{k !} \prod_{m=0}^{p-1}\left(\beta+k-q-3-\alpha \sigma_{m+1}\right) \prod_{m=p}^{q}(k+p-m)-\sum_{j=\alpha}^{k-1} \frac{C_{j}}{(k-j) !} \\
(\alpha \leqq k \leqq q+1) .
\end{array}
$$

In particular,

$$
\text { (4.11) } \quad E_{q+1}=1 ; \quad E_{q}=(q+1)(\beta-p-2)-\alpha \sum_{m=0}^{q} \rho_{m}+q(q+1) / 2
$$

and

$$
C_{q+1}=1 ; \quad C_{q}=p(\beta-q-3)-\alpha \sum_{m=1}^{p} \sigma_{m}+\alpha(1-\alpha) / 2+q(q+1) / 2 .
$$

Here $\alpha$ and $\beta$ are the quantities defined by (2.2).

Proof. The differential equation $L(y)=0$ may be written in the form

$$
\begin{aligned}
t^{\alpha} \prod_{m=0}^{q}(\theta+\beta-p & \left.-2-\alpha \rho_{m}\right) y(t) \\
& =\prod_{m=0}^{p-1}\left(\theta+\beta-q-3-\alpha \sigma_{m+1}\right) \prod_{m=p}^{q}(\theta+p-m) y(t) .
\end{aligned}
$$

In Lemma 4.1 put $B_{m}=\beta-p-2-\alpha \rho_{m}$ and obtain the following representa- 
tion for the left side of (4.13):

$$
t^{\alpha} \sum_{k=0}^{q+1} E_{k} t^{k} \frac{d^{k}}{d t^{k}} y(t)
$$

where $E_{k}(k=0,1, \cdots, q+1)$ is given by (4.9). If we put $B_{m}=\beta-q-3-\alpha \sigma_{m+1}$ for $m=0,1, \cdots, p-1$ and $B_{m}=p-m$ for $m=p, \cdots, q$, then from Lemma 4.1 the right side of (4.13) takes the form

$$
\sum_{k=0}^{q+1} C_{k} t^{k} \frac{d^{k}}{d t^{k}} y(t)
$$

where

$$
\begin{array}{r}
C_{k}=\frac{1}{k !} \prod_{m=0}^{p-1}\left(\beta+k-q-3-\alpha \sigma_{m+1}\right) \prod_{m=p}^{q}(k+p-m)-\sum_{j=0}^{k-1} \frac{C_{j}}{(k-j) !} \\
(0 \leqq k \leqq q+1) .
\end{array}
$$

By recursion $C_{k}=0$ for $k=0,1, \cdots, \alpha-1$ and hence this latter formula reduces to (4.10). From (4.14) and (4.15) we have

$$
t^{\alpha} \sum_{k=0}^{q+1} E_{k} t^{k} y^{(k)}(t)=\sum_{k=\alpha}^{q+1} C_{k} t^{k} y^{(k)}(t)
$$

which yields (4.8). Finally, relations (4.11) and (4.12) are a consequence of (4.6) with the above choices of $B_{m}$.

5. Properties of the differential equation. Let the variable $t$ in the differential equation $L(y(t))=0$ be extended to the complex plane. From (4.8) we see that $L(y)=0$ is of Fuchsian type [5]. If $p=0$ the regular singular points of $L(y)=0$ are $t=\infty$ and the $\alpha$ roots of unity for which $t^{\alpha}=1$. If $p \geqq 1$ the regular singular points are $t=0, t=\infty$ and the $\alpha$ roots of unity for which $t^{\alpha}=1$. In any case, there are no singularities for $t$ real with $0<t<1$.

The point $t=1$ is a regular singularity of $L(y)=0$ for each $p \leqq q$. From (4.8) we see that the indicial equation of the differential equation at this point is

$$
\mu(\mu-1) \cdots(\mu-q)+\frac{E_{q}-C_{q}}{\alpha} \mu(\mu-1) \cdots(\mu-q+1)=0
$$

which has the solutions

$$
\mu=0,1, \cdots, q-1 \text { and } \mu=q+2 .
$$

Here we have used the relation $C_{q}-E_{q}=2 \alpha$.

When $p=0$ the point $t=0$ is an ordinary point of $L(y)=0$. If $p \geqq 1$ then $t=0$ is a regular singularity of the differential equation and from (4.3) it follows that the indicial equation at the origin is 


$$
\prod_{k=1}^{p}\left(\mu+\beta-q-3-\alpha \sigma_{k}\right) \prod_{r=0}^{\alpha-1}(\mu-r)=0
$$

which has the solutions

$$
\mu=0,1, \cdots, \alpha-1 ; \quad \mu=-\beta+q+3+\alpha \sigma_{k} \quad(1 \leqq k \leqq p) .
$$

The function $\phi(t)$ has been defined by (3.2) for $t$ real with $0<t \leqq 1$. Since $\phi(t)$ solves $L(y)=0$ for $0<t \leqq 1$ it follows from the location of the singularities of the differential equation that $\phi(t)$ is analytic for $0<t<1$. Hence, $\phi(t)$ may be extended analytically and the extension is regular except for possible branch points at the regular singular points of $L(y)=0$.

If we let $K$ denote the distance from $t=1$ to the nearest of the other singularities of $L(y)=0$, then it follows from the theory of Frobenius [5] and (5.1) that the functions

$$
\begin{aligned}
y_{0}(t) & =(1-t)^{q+2} \psi_{0,0}(t) y_{k}(t) \\
& =(1-t)^{q-k} \sum_{m=0}^{k}[\log (t-1)]^{m} \psi_{k, m}(t) \quad(1 \leqq k \leqq q),
\end{aligned}
$$

form a fundamental system of solutions of $L(y)=0$ in the region $|t-1|<K$. Here the functions $\psi_{k, m}(t)$ are regular for $|t-1|<K$ and $\psi_{k, 0}(1) \neq 0$ for $k=0$, $1, \cdots, q$. Since $\phi(t)$ is an integral of $L(y)=0$ in the neighborhood of $t=1$

$$
\phi(t)=\sum_{k=0}^{q} A_{k} y_{k}(t), \quad|t-1|<K,
$$

where the $A_{k}$ are constants. From Theorem 3.1 we have that $\phi(t)=O\left((1-t)^{q+2}\right)$ as $t \rightarrow 1$ through positive values of $t$. Since $\psi_{k, 0}(1) \neq 0$ it follows that $A_{k}=0$ for $k=1, \cdots, q$ and hence $\phi(t)$ is a multiple of $y_{0}(t)$. Hence, by Taylor's theorem,

$$
\phi(t)=\sum_{m=0}^{\infty} \frac{\phi^{(q+2+m)}(1)}{\Gamma(q+3+m)}(t-1)^{q+2+m}
$$

for values of $t$ such that $|t-1|<K$.

The results of this section may be formulated in the following theorem.

TheOREM 5.1. Let the variable $t$ in the differential equation (4.3) be extended to the complex domain. Then $L(y(t))=0$ is of Fuchsian type. If $p=0$ the regular singular points are $t=\infty$ and the $\alpha$ roots of unity for which $t^{\alpha}=1$. If $p>0$ then $t=0$ is also a regular singular point of $L(y(t))=0$. If $K$ denotes the distance from $t=1$ to the nearest of the other singularities of the differential equation, then $\phi(t)$ has the Taylor series expansion (5.3) for values of $t$ such that $|t-1|<K$.

6. Identification of the constants. In this section we shall see that $\phi(t)$ acts as a generating function for the constants $c_{m}$. To show this we shall first need the following result. 
Lemma 6.1. Let $m$ be any non-negative integer and let $\lambda_{0}$ be defined by (3.1). Then

$$
\lim _{t \rightarrow 0} t^{x+m} \phi^{(m)}(t)=0
$$

provided $\operatorname{Re}(x)>\lambda_{0}$.

Proof. $\phi(t)$ satisfies $L(y)=0$ which has $t=0$ as an ordinary point if $p=0$ and a regular singular point if $p>0$. The assertion is trivially true for $p=0$ since $\lambda_{0} \geqq 0$. If $p>0$ it follows from the theory of Frobenius that $\phi(t)$ admits the expansion

$$
\phi(t)=\sum_{i}(\log t)^{k i} t^{\mu} \sum_{n=0}^{\infty} d_{i, n} t^{n} \quad\left(d_{i, 0} \neq 0\right),
$$

for $|t|<1$. From (5.2) and (3.1) we have that

$$
\operatorname{Re}\left(\mu_{i}\right) \geqq-\lambda_{0} \text { for all } i \text {. }
$$

Consequently, for any non-negative integer $m$,

$$
\phi^{(m)}(t)=O\left(t^{-\lambda-m}\right)
$$

as $t \rightarrow 0$, which proves the assertion.

THEOREM 6.1. The constants $c_{m}(m=0,1, \cdots)$ occurring in the asymptotic factorial expansion of $g_{q}(w)$ are precisely the constants which occur in the Taylor series expansion of $\phi(t)$ about $t=1$. In fact,

$$
c_{m}=(-1)^{m+q} \phi^{(q+2+m)}(1) \quad(m \geqq 0) .
$$

Proof. Integration of (3.3) by parts $N+1$ times yields

$$
H(x)=\sum_{n=0}^{N} \frac{(-1)^{n} \Gamma(x)}{\Gamma(x+n+1)}\left[\phi^{(n)}(t) t^{x+n}\right]_{0}^{1}+\frac{(-1)^{N+1} \Gamma(x)}{\Gamma(x+N+1)} \int_{0}^{1} t^{x+N} \phi^{(N+1)}(t) d t,
$$

provided $\operatorname{Re}(x)>\lambda_{0}$. From Lemma 6.1 we have that

$$
\left[\phi^{(n)}(t) t^{x+n}\right]_{0}^{1}=\phi^{(n)}(1)
$$

for $\operatorname{Re}(x)>\lambda_{0}$. Since $\phi^{(n)}(1)=0$ for $n=0,1, \cdots, q+1$, by (5.3), we may set $n=m+q+2$ and obtain

$$
H(x)=\sum_{m=0}^{M} \frac{(-1)^{q+2+m} \Gamma(x)}{\Gamma(x+q+3+m)} \phi^{(q+2+m)}(1)+R_{M},
$$

provided $\operatorname{Re}(x)>\lambda_{0}$. Here $M$ is any non-negative integer and 


$$
R_{M}=\frac{(-1)^{q+3+M} \Gamma(x)}{\Gamma(x+q+4+M)}\left\{\phi^{(q+3+M)}(1)-\int_{0}^{1} t^{x+q+3+M} \phi^{(q+4+M)}(t) d t\right\} .
$$

Let $x$ tend to infinity along the positive real axis. Then the integral is of $O(1)$ and consequently

$$
R_{M}=O(\Gamma(x) / \Gamma(x+q+4+M)) .
$$

The relation (6.1) now follows by comparing (6.2) with (2.6). For, the coefficients in (2.6) are already uniquely determined if we require (2.6) only for $x$ real and positive.

7. The recursion formula. We substitute (6.1) into (5.3) and obtain

$$
\phi(t)=\sum_{m=0}^{\infty} \frac{(-1)^{m+q} C_{m}}{\Gamma(q+3+m)}(t-1)^{q+2+m} \quad|t-1|<K .
$$

Since $\phi(t)$ is an integral of the differential equation $L(y)=0$ we may determine a recursion formula for the constants $c_{m}$ by substituting (7.1) into (4.8). The formula provides a method of computing the constants since we know that $c_{0}=1$.

Theorem 7.1. Let the constants $C_{k}(k=\alpha, \alpha+1, \cdots, q+1)$ and $E_{k}$ $(k=0,1, \cdots, q+1)$ be as defined in the formulation of Theorem 4.2. The constants $c_{m}(m=0,1, \cdots)$, defined by Theorem 2.1 , satisfy the following recursion formula of length $q$ :

$$
c_{0}=1
$$

and

$$
c_{m}=(m+2) !(\alpha m)^{-1} \sum_{i=1}^{q} a_{m i} c_{m-i},
$$

$\left(c_{-1}=c_{-2}=\cdots=0\right)$, where

$$
a_{m i}=(-1)^{i-1} \sum_{k=q-i}^{q+1}\left(\begin{array}{c}
k \\
q-i
\end{array}\right) E_{k} /(m+q+2-i-k) !
$$

if $p \leqq i \leqq q$, while

$$
\begin{aligned}
a_{m i}=(-1)^{i-1} \sum_{k=q-i}^{q+1}\left\{\left(\begin{array}{c}
k \\
q-i
\end{array}\right) E_{k}\right. & \left.\quad-\left(\begin{array}{c}
k-\alpha \\
q-i-\alpha
\end{array}\right) C_{k}\right\} /(m+q+2-i-k) !
\end{aligned}
$$

if $1 \leqq i \leqq p-1$. Note that the last formula and, hence, the $C_{k}$ are of no importance if $p=0$ or $p=1$. 
Proof. Let us transform the independent variable in (4.8) by the relation $t=t^{\prime}+1$ and then drop the prime. We obtain the differential equation

$$
\sum_{k=\alpha}^{q+1}\left\{E_{k}(t+1)^{k}-C_{k}(t+1)^{k-\alpha}\right\} y^{(k)}(t)+\sum_{k=0}^{\alpha-1} E_{k}(t+1)^{k} y^{(k)}(t)=0
$$

which is satisfied by

$$
y(t) \equiv \phi(t+1)=\sum_{m=0}^{\infty} \frac{(-1)^{m+q} c_{m}}{\Gamma(q+3+m)} t^{q+2+m}
$$

in the neighborhood of $t=0$. Employing the binomial theorem, we may write the differential equation in the form

$$
\sum_{j=0}^{\alpha-1} \sum_{k=j}^{q+1}\left(\begin{array}{l}
k \\
j
\end{array}\right) E_{k} t^{k-j} y^{(k)}(t)+\sum_{j=\alpha}^{q+1} \sum_{k=j}^{q+1}\left\{\left(\begin{array}{c}
k \\
j
\end{array}\right) E_{k}-\left(\begin{array}{c}
k-\alpha \\
j-\alpha
\end{array}\right) C_{k}\right\} t^{k-j} y^{(k)}(t)=0 .
$$

By (7.5) the coefficient of $t^{m+2}$ in $t^{k-j} y^{(k)}(t)$ is equal to

$$
(-1)^{m+j} c_{m+j-q} /(m+2-j-k) !
$$

mplying that, for each integer $m$,

$$
\begin{aligned}
& \sum_{j=0}^{\alpha-1}(-1)^{m+j} c_{m+j-q} \sum_{k=j}^{q+1}\left(\begin{array}{l}
k \\
j
\end{array}\right) E_{k} /(m+2+j-k) ! \\
& \quad+\sum_{j=\alpha}^{q+1}(-1)^{m+i} c_{m+j-q} \sum_{k=j}^{q+1}\left\{\left(\begin{array}{l}
k \\
j
\end{array}\right) E_{k}-\left(\begin{array}{c}
k-\alpha \\
j-\alpha
\end{array}\right) C_{k}\right\} /(m+2+j-k) !=0,
\end{aligned}
$$

provided we make the agreement that $c_{m}=0$ for $m<0$. From (4.11) and (4.12), the coefficients of $c_{m+1}$ and $c_{m}$ in the latter formula are 0 and

$$
(-1)^{m+q} \alpha m /(m+2) \text { ! }
$$

respectively. Hence,

$$
\begin{aligned}
c_{m}= & \frac{(m+2) !}{\alpha m}\left[\sum_{j=0}^{\alpha-1}(-1)^{j+q+1} c_{m+j-q} \sum_{k=j}^{q+1}\left(\begin{array}{l}
k \\
j
\end{array}\right) E_{k} /(m+2+j-k) !\right. \\
& \left.+\sum_{j=\alpha}^{q-1}(-1)^{j+q+1} c_{m+j-q} \sum_{k=j}^{q+1}\left\{\left(\begin{array}{l}
k \\
j
\end{array}\right) E_{k}-\left(\begin{array}{l}
k-\alpha \\
j-\alpha
\end{array}\right) C_{k}\right\} /(m+2+j-k) !\right] .
\end{aligned}
$$

This formula reduces to (7.2) if we set $i=q-j$ and introduce the notations (7.3) and (7.4).

8. Several examples. As a first illustration let us consider the coefficient function ${ }_{0} g_{1}(w)$. In the special case where $\rho_{0}=1{ }_{0} g_{1}(w)$ reduces to the coefficient function of the Bessel function. Here $p=0, q=1, \alpha=2$ and $\beta=\rho_{0}+\rho_{1}$ $-1 / 2 ;(7.2)$ reduces to 


$$
c_{m}=c_{m-1}(m+2) !(2 m)^{-1} \sum_{k=0}^{2} E_{k} /(m+2-k) !, \quad(m \geqq 1) .
$$

From (4.11) we recursively calculate

$$
E_{0}=\left(\rho_{1}-\rho_{0}-5 / 2\right)\left(\rho_{0}-\rho_{1}-5 / 2\right) ; \quad E_{1}=-4 ; \quad E_{2}=1 .
$$

Hence,

$$
\begin{aligned}
c_{m} & =\frac{c_{m-1}}{2 m}\left[(m+1)(m+2)-4(m+2)-\left(\rho_{1}-\rho_{0}\right)^{2}+25 / 4\right] \\
& =\frac{c_{m-1}}{8 m}\left[(2 m-1)^{2}-4\left(\rho_{1}-\rho_{0}\right)^{2}\right],
\end{aligned}
$$

Since $c_{0}=1$ it follows that

$$
c_{m}=\frac{1}{2^{3 m} m !} \prod_{k=1}^{m}\left[(2 k-1)^{2}-4\left(\rho_{1}-\rho_{0}\right)^{2}\right] \quad(m \geqq 0) .
$$

When $\rho_{0}=1$ this reduces to the familiar expression for the coefficients in the asymptotic expansion of the Bessel function [8, p. 368].

Theorem 7.1 also yields the explicit expression for the constants in the factorial expansion of ${ }_{1} g_{1}(w)$. In this case one obtains

$$
c_{m}=\frac{1}{m !} \sum_{k=0}^{m-1}\left(\rho_{0}-\sigma_{1}+k\right)\left(\rho_{1}-\sigma_{1}+k\right), \quad(m \geqq 0) .
$$

If we set $\rho_{0}=1$ these reduce to the coefficients which occur in the asymptotic expansion of the confluent hypergeometric function [8, p. 342].

As a final illustration let us consider the coefficient function ${ }_{2} g_{2}(w)$. In this case $p=q=2, \alpha=1$ and $\beta=\rho_{0}+\rho_{1}+\rho_{2}-\sigma_{1}-\sigma_{2} ;(7.2)$ reduces to

$$
\begin{aligned}
c_{m}= & \frac{(m+2) !}{m}\left[c_{m-1} \sum_{k=1}^{3}\left\{k E_{k}-C_{k}\right\} /(m+3-k) !\right. & \\
& \left.-c_{m-2} \sum_{k=0}^{3} E_{k} /(m+2-k !)\right], & (m \geqq 1) .
\end{aligned}
$$

Here the constants $E_{k}$ and $C_{k}$ are given by Theorem 4.2. The recursion formula for $c_{m}$ thus depends on the last two previously calculated coefficients since $q=2$. Consequently, we cannot write the general explicit formula for $c_{m}$. For specified values of $\rho_{j}$ and $\sigma_{i}$, however, there is no difficulty as may be illustrated by a numerical example.

Suppose $\rho_{0}=1, \rho_{1}=2.2-0.5 i, \rho_{2}=2.2+0.5 i, \sigma_{1}=0.6$ and $\sigma_{2}=-0.2$. Then $\beta=5$ and Theorem 4.2 yields $E_{0}=1, E_{1}=0.29, E_{2}=0.6, E_{3}=1, C_{1}=0.48$, $C_{2}=2.6$ and $C_{3}=1$. From (8.3) we calculate 


$$
\begin{aligned}
& c_{0}=1, \\
& c_{1}=7.61 c_{0}=7.61, \\
& c_{2}=9.105 c_{1}-8.98 c_{0}=60.30905 \\
& c_{3}=10.9367 c_{2}-24.4833 c_{1}=473.2816, \text { etc. }
\end{aligned}
$$

\section{BIBLIOGRAPHY}

1. G. Doetsch, Theorie und Anwendung der Laplace-Transformation, Dover Publications, 1943.

2. W. B. Ford, The asymptotic developments of functions defined by Maclaurin series, University of Michigan Studies, Scientific Series, vol. 11, 1936.

3. H. K. Hughes, On the asymptotic expansion of entire functions defined by Maclaurin series, Bull. Amer. Math. Soc. vol. 50 (1944) pp. 425-430.

4. - The asymptotic developments of a class of entire functions, Bull. Amer. Math. Soc. vol. 51 (1945) pp. 456-461.

5. E. L. Ince, Ordinary differential equations, Dover Publications, 1926.

6. J. H. B. Kemperman, Unpublished notes for a seminar first presented at Purdue University in 1952 .

7. T. D. Riney, On the coefficients in asymptotic factorial expansions, Proc. Amer. Math. Soc. vol. 7 (1956) pp. 245-249.

8. E. T. Whittaker and G. H. Watson, $A$ course of modern analysis, Cambridge University Press, 1952.

9. E. M. Wright, The asymptotic expansion of integral functions defined by Taylor series, Philos. Trans. Roy. Soc. London, Series A, vol. 238 (1940) pp. 423-451.

10. - The asymptotic expansion of integral functions defined by Taylor series, Philos. Trans. Roy. Soc. London, Ser. A vol. 239 (1941) pp. 217-232.

11. - The asymptotic expansion of the generalized hypergeometric function, Proc. London Math. Soc. (2) vol. 46 (1940) pp. 389-408.

Bell Telephone Laboratories, Allentown, Pa.

Purdue University,

LAFAYETTE, IND. 